\title{
Research on Suppliers' Performance Clustering Method with the Contracts Evaluation Data
}

\author{
Lidong Liu ${ }^{1}$, Wengbing Chang ${ }^{2}$ and Shenghan Zhou ${ }^{2, *}$ \\ ${ }^{1}$ School of Economics and Management, Beihang University, Beijing, China 100191 \\ ${ }^{2}$ School of Reliability and System Engineering, Beihang University, Beijing, China 100191 \\ ${ }^{*}$ Corresponding author
}

\begin{abstract}
- this paper proposed suppliers' performance clustering method with the re-analysis on contracts evaluation data. The study summarizes the basic definitions of the equipment manufacturing industry evaluation methods. Then the k-means clustering method based on the data re-analysis was defined. Finally the study gives an empirical example to present the main process of the proposed clustering method with 422 contracts evaluation data. The result shows that it is feasible.
\end{abstract}

\section{Keywords-clustering method; $k$-means; supplier performance}

\section{INTRODUCTION}

The supplier performance management problem plays an important role in equipment $\mathrm{R} \& \mathrm{D}$ contracts management. The decision makers develop many evaluation tools to analyze the suppliers' contracts performance and get many results from different evaluation works. However, the too many report data cause another problem: how to discover previously unknown and hidden knowledge from evaluation data sources?

With the rapid developments of evaluation method, the data analysis is usually applied to re-analysis the evaluation result and got the further conclusion. It is also a question that how to balance the equipment suppliers' performance and its profit to strengthen the mechanism construction.

The comprehensive evaluation is of great significance to the improvement of product quality. The $k$-means algorithm ${ }^{[1]}$, which uses the distance as the evaluation index of similarity, is a typical clustering algorithm. Because of the advantages of quick, simple, high efficiency for large data sets and scalability, it is widely used in many domains. For example, social networks ${ }^{[2]}$, image processing ${ }^{[3]}$, android malware detection ${ }^{[4]}$. Cornell analyzed the American space industry's structure as well as its approach to innovation and to the globalization of the space sector ${ }^{[5]}$. Yang discussed the implementation of manufacturing practices in terms of production planning, quality management, human resource management, and capacity management ${ }^{[6]}$. In the past, the evaluation of contracts performance mainly used the method of the subjective ratings of experts ${ }^{[7]}$. The matter that the quantitative results are not in conformity with the qualitative analysis will not appear ${ }^{[8]}$. To solve these problems, Kai Meng Tay and Chee Peng Lim proposed Fuzzy Inference Techniques in analysis methodology ${ }^{[9][10]}$. Galvao etc. developed a new nonparametric quantile regression estimation method with min-distance ${ }^{[11]}$. And Monte Carlo simulation method is proved that the improved test method performs better than traditional test methods in Large-scale example ${ }^{[12]}$.

The paper describes the basic definition of mean, variance and correlation coefficient in section 1. Then the paper introduced the main processes to make the normality test with Shapiro-Wilk (SW) statistics. Then, in section 2, we put forward a performance clustering method combined with data test and k-means algorithm. The proposed method is used for the quantitative evaluation of equipment contracts. In section 3 , we use an empirical case to verify the feasibility of the model which is established in this paper. Finally, in section 4, we conclude the study.

\section{BASIC DEFINITION}

Definition1 the mean of the data set is:

$$
\bar{X}=\frac{\sum_{i=1}^{n} X_{i}}{n}
$$

The $\mathrm{X}_{i}$ shows the evaluated projects, and the $n$ is the number of the evaluated projects.

Definition2 the variance of the data set is the measure of its discrete degree. The variance $\mathrm{S}$ is defined as:

$$
S^{2}=\frac{1}{n-1} \sum_{i=1}^{n}\left(x_{i}-\bar{x}\right)^{2}
$$

Definition 3 the Pearson correlation coefficient is defined as follow.

Set two random variables set is $(X Y)$, the variance is $\operatorname{var}(X)$ and $\operatorname{var}(Y)$, their covariances is $\operatorname{cov}(X, Y)$. The correlation coefficient is:

$$
\rho_{X Y}=\operatorname{Corr}(X, Y)=\frac{\operatorname{Cov}(X, Y)}{\sqrt{\operatorname{Var}(X)} \sqrt{\operatorname{Var}(Y)}}
$$

Include,

$$
\operatorname{Cov}(X, Y)=E[X-E(X)][Y-E(Y)]=E(X Y)-E(X) \cdot E(Y)
$$




$$
E(X)=\frac{\sum_{i=1}^{n} X_{i}}{n} \quad E(Y)=\frac{\sum_{i=1}^{n} Y_{i}}{n}
$$

Set $\left(X_{1}, Y_{1}\right),\left(X_{2}, Y_{2}\right), \cdots,\left(X_{n}, Y_{n}\right)$ is the sample of $(X, Y)$, he correlation coefficient of sample is

$$
r_{X Y}=\frac{S_{X Y}^{2}}{\sqrt{S_{X}^{2}} \times \sqrt{S_{Y}^{2}}}=\frac{\sum_{i=1}^{n}\left(X_{i}-\bar{X}\right)\left(Y_{i}-\bar{Y}\right)}{\sqrt{\sum_{i=1}^{n}\left(X_{i}-\bar{X}\right)^{2}} \sqrt{\sum_{i=1}^{n}\left(Y_{i}-\bar{Y}\right)^{2}}}
$$

Include,

The $\overline{\boldsymbol{X}}$ and $\overline{\boldsymbol{Y}}$ is the expectation of $(X, Y)$, the $S_{X}^{2}$ and $S_{Y}^{2}$ is the sample variance of $\mathrm{X}$ and $\mathrm{Y}$, the $S_{X Y}^{2}$ is the sample covariance of $(X, Y)$.

The processes of Shapiro-Wilk (SW) test include two steps.:

(1) Order the observations of independent samples $S_{i}(i=1,2, \ldots, N)$, get the $S_{1}<S_{2}<\ldots, S_{N}$ as vector $S^{T}=\left(S_{1}, S_{2, \ldots}, S_{N}\right)$. The $S^{T}$ is the transposed vector of $S_{i}$

Hypothesis:

$\mathrm{H}_{0}$ : there were no significant differences between sample data and normal distribution

$\mathrm{H}_{1}$ : there were significant differences between sample data and normal distribution

(2) the test statistics $W$ is defined as.

$$
W=\frac{\left(\sum_{i=1}^{N} a_{i} s_{i}\right)^{2}}{\sum_{i=1}^{N}\left(s_{i}-\bar{s}\right)^{2}}
$$

Include,

$$
\bar{s}=\frac{1}{N} \sum_{i=1}^{N} s_{i}
$$

The $W \in[0,1]$, the sample near normal distribution while $W$ near 1 . And that didn't coincided with normal distribution while $W$ near 0 .

\section{SUPPLIERS CluStering WITH K-MEANS ALGORITHM}

The paper adopt $k$-means clustering method to evaluate the suppliers with the report data. $k$-means clustering algorithm is a typical hard clustering algorithm. The method usually uses the error square sum function as the objective function of the optimization. The error square sum function is defined as follows:

$$
F=\sum_{i=1}^{K} \sum_{x \in S_{i}}\left\|x-\mu_{i}\right\|^{2}
$$

Here, $K$ represents the number of clusters. $S_{i}(i=1,2, \ldots, K)$ is a cluster. $x$ represents a data object in the cluster $S_{i}$. $\mu_{i}$ is the mean value of the cluster $S_{i}$.

Obviously, $F$, whose value depends on the $K$ cluster center pointer, represents the error square sum between the data samples and the cluster centers. The smaller the $F$, the better the quality of the clustering results.

There are three main methods to selection the initial cluster center points.

(1) The authorities designate the initial center points by experience. The method general applied to the easy cluster problems. The authorities have significant experience in the evaluation problem.

(2) The projects were divided into k classes randomly. The mean of every class was defined the initial center.

(3) the max-min principle. Assume the projects were divided into $\mathrm{k}$ classes, and the centers of two max-distance classes is $x_{i 1}, x_{i 2}$, the distance between them $d\left(x_{i 1}, x_{i 2}\right)=\max \left(d_{i j}\right)$. Find the $x_{i 3}$, center, it Meets $\min \left\{d\left(x_{i 3}, x_{i 1}\right), d\left(x_{i 3}\right.\right.$, $\left.\left.x_{i 2},\right)\right\}=\max \left\{\min \left[d\left(x_{j} x_{i 1},\right), d\left(x_{j}, x_{i 2},\right), i \neq i_{1}, i_{2}\right]\right\}$, then finext center point until we find all the $\mathrm{k}$ centers. There are some main points to consider when using k-means algorithm to cluster the evaluation objects:

(1) Select a certain distance as the similarity measure between the data samples.

(2) Select a criterion function to evaluate the clustering performance.

(3) The computation of similarity is based on the average value of the objects in a cluster.

The study defines the distance as follows. Suppose that $\mathrm{N}$ times landing is divided into $K$ class, namely $R_{1}, R_{2}, \cdots, R_{i}, \cdots, R_{j}$, $R_{K}$. $n_{i}$ represents the number of landings in $R_{i}$, and $\overline{R_{i}}$ represents the center of $R_{i}$.

$$
\overline{R_{i}}=\frac{1}{n} \sum_{U_{i} \in G_{i}} U_{i}
$$

The diameter of the class $R_{i}$ is given by the formula (6).

$$
D\left(R_{i}\right)=\sum_{U_{i} \in R_{i}} D\left(U_{i}, \overline{R_{i}}\right)=\sum_{U_{i} \in R_{i}} \sqrt{\left(U_{i}^{1}-\overline{R_{i}^{1}}\right)^{2}+\left(U_{i}^{2}-\overline{R_{i}^{2}}\right)^{2}+\cdots+\left(U_{i}^{P}-\overline{R_{i}^{P}}\right)^{2}}
$$

The distance between classes $R_{i}$ and $R_{j}$ is given by the formula (7).

$$
D\left(R_{i}, R_{j}\right)=D\left(\overline{R_{i}}, \overline{R_{j}}\right)=\sqrt{\left(R_{i}^{1}-\overline{R_{j}^{1}}\right)^{2}+\left(R_{i}^{2}-\overline{R_{j}^{2}}\right)^{2}+\cdots+\left(R_{i}^{P}-\overline{R_{j}^{P}}\right)^{2}}
$$

The process of $K$-means algorithm can be described as: 
Step 1, assuming that there are $n$ objects in the database and the number of clusters is $K$. Determine an initial cluster center for each cluster, so there are $K$ initial cluster centers $L^{(0)}=\left\{x_{1}^{(0)} x_{2}^{(0)}, \ldots x_{k}^{(0)}\right\}$.

$$
\text { Set } G_{i}^{(0)}=\left\{x \mid d\left(x, x_{i}^{(0)} \leq d\left(x, x_{j}^{(0)}\right), j=1,2 \ldots, k, j \neq i\right\}, i=1,2, \ldots, k\right.
$$

The projects were divided into $\mathrm{k}$ disjoint classes $G_{i}^{(0)}$, $\mathrm{i}=1,2, \ldots \mathrm{k}$. And the initial classes is $G^{(0)}=\left\{G_{1}^{(0)}, G_{2}^{(0)}, \ldots G_{k}^{(0)}\right\}$

Step 2, the samples are distributed to the nearest neighbor clustering according to the principle of minimum distance. The improved class center is $L^{(1)}=\left\{x_{1}^{(1)}, x_{2}^{(1)}, \ldots x_{k}^{(1)}\right\}$, and the $x_{i}^{(1)}=\frac{1}{n_{i}} \sum_{x_{i} \in G_{i}^{(0)}} x_{i}, i=1,2, \ldots, k$.

Step 3, using the sample mean in each cluster as a new cluster center and the step $\mathrm{m}$ get the improved classed $G^{(m)}=\left\{G_{1}^{(m)}, G_{2}^{(m)}, \ldots G_{k}^{(m)}\right\}$

Step 4, Repeat step 2 and 3 until the clustering center no longer change, and then output $K$ clusters.

\section{EMPIRICAL RESEARCH}

The data of this study comes from an equipment manufacture company. And they have many suppliers in the past ten years. Some different evaluation reported for performance. The board thinks the result data is too complex to classify the supplier performance. And they even think the existing evaluation system may mislead the supplier management work.

The below calculation process takes 422 contracts performance evaluation report scores as an example. These contracts include three main types, customization contracts, product contracts and research contracts. All of these contracts were evaluated in a unified framework. The initial evaluation results were modified by different group with the compensation scores. The compensation score is given by different experts. The final scores are got through a special algorithm. The board think the compensation process may be useless. And they also want to know which contracts and suppliers are really performed well as expected.

\section{A. Descriptive Analysis}

The template is designed so that author affiliations are not repeated each time for multiple authors of the same affiliation. Please keep your affiliations as succinct as possible (for example, do not differentiate among departments of the same organization). This template was designed for two affiliations.

The comparison of initial and final scores is shown in Figure 1. The difference between the min and max score is 5.03, accounting for about 5 percent of average initial scores, it plays an insignificant role in final scores. On the other hand, it suggests the compensation system donot work as it been designed.

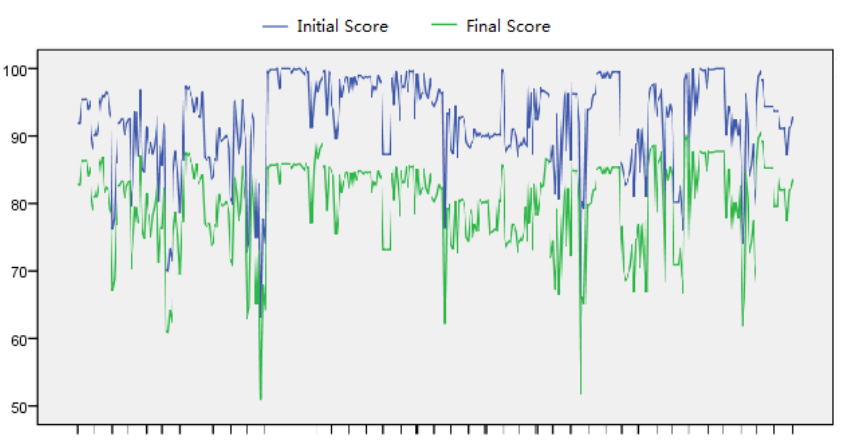

FIGURE I. COMPARISON OF INITIAL AND FINAL SCORES

The pareto diagram of 422 contracts' initial scores is given in Figure 2. The curve suggests the contracts from same supplier trend to get the closed scores.

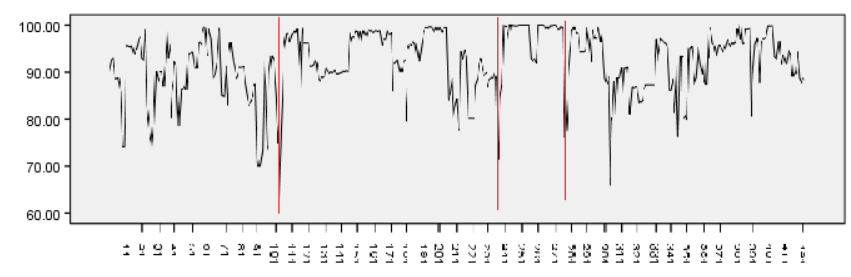

FIGURE II. 422 CONTRACTS' PARETO DIAGRAM

Another problem takes place in the situation while the evaluation experts group changes as the red line in figure 2 . These breakpoints suggest the different tendencies of different expert groups. Sometimes the contracts scores may be distorted. Eg. The contracts from 239 to 276 got very high scores.

\section{B. Correlational Analysis}

The further study makes correlational analyses of the initial scores, compensation scores and final scores. The result was given as follow Table I. The significance level $\alpha=0.01$.

TABLE I. CORRELATIONAL ANALYSES OF SCORES

\begin{tabular}{lccc}
\hline & Initial & Compensation & Final \\
\hline Initial & 1 & & \\
Compensation & $-0.326^{*}$ & 1 & \\
Final & $0.946^{*}$ & -0.298 & 1 \\
\hline
\end{tabular}

The table shows the correlation coefficient of initial and compensation scores is -0.326 . It is significant negative correlation. And the relationship between initial and final scores is significant positive correlation.

According to our statistics, there are $72.8 \%$ suppliers which got more than 98 initial score been modified with -14.13 compensation scores or more. The compensation level is about 3 times as average level. The result support suspicion, the compensation experts trend to make balance by giving greater modification to higher scores. 


\section{Normality Test}

The initial and final scores probability distribution diagram are given in Figure 3 and 4. The study makes the KolmogorovSmirnova test and Shapiro-Wilk test. The result is reported in table II.

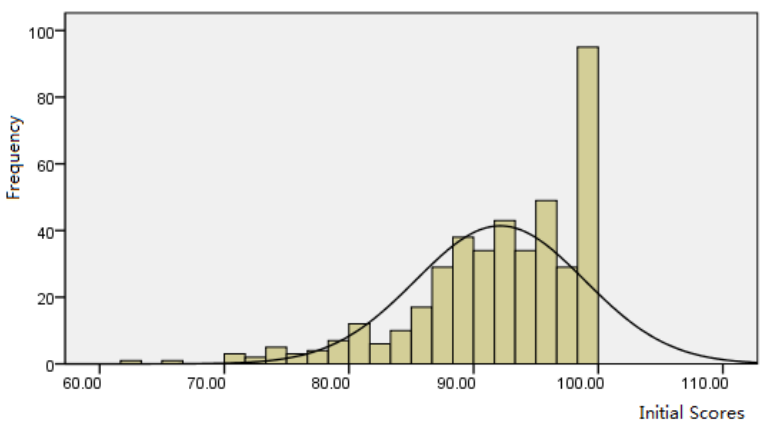

FIGURE III. INITIAL SCORES PROBABILITY DISTRIBUTION

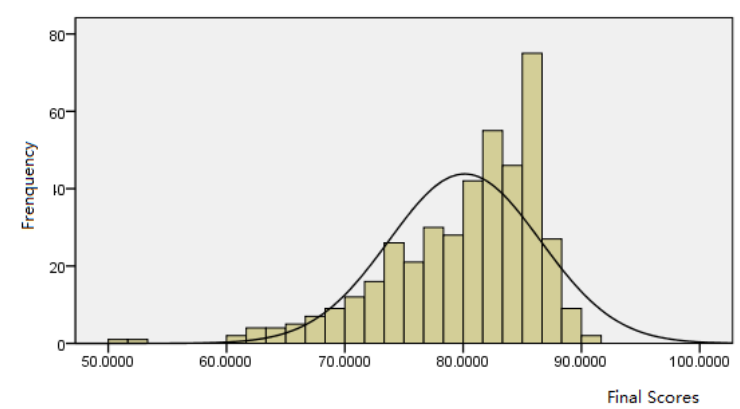

FIGURE IV. FINAL SCORES PROBABILITY DISTRIBUTION

TABLE II. NORMALITY TEST RESULT

\begin{tabular}{cccccc}
\hline \multirow{2}{*}{ Test } & \multicolumn{2}{c}{ Kolmogorov-Smirnova } & & \multicolumn{2}{c}{ Shapiro-Wilk } \\
\cline { 2 - 3 } & Statistics & Sig. & Statistics & Sig. \\
\hline Initial & 0.123 & 0.000 & 0.908 & 0.000 \\
Final & 0.113 & 0.000 & 0.915 & 0.000
\end{tabular}

The results show the initial and final scores don't coincided with normal distribution. The data suggest the expert trend to give a higher score and the compensation process donot work well. The suspicion of board is reasonable hypothesis.

\section{Comprehensive Performance Clustering}

The suppliers' comprehensive performance is related with many causes. So the study makes the $k$-means clustering analysis according to the contracts type. The sample contracts include three types, customization, product and research.

The comprehensive performance clustering results are shown in Table III. The first cluster means the better product capability. The third cluster presents the suppliers who have better research capability. The second column includes the other suppliers.
TABLE III. FINAL CLUSTERING CENTERS

\begin{tabular}{lccc}
\hline & \multicolumn{3}{c}{ Clustering } \\
\cline { 2 - 4 } & 1 & 2 & 3 \\
\hline Customization Contracts & 90.7830 & 0.0000 & 88.0970 \\
Product Contracts & 88.4430 & 85.1042 & 85.2936 \\
Research Contracts & 0.0000 & 91.5955 & 82.7840 \\
\hline
\end{tabular}

\section{CONCLUSION}

This paper attempts to establish a performance clustering method combined with data test and k-means for the quantitative evaluation of equipment contracts. And compared with the traditional method for evaluating the performance of suppliers, the main advantages of this research are as following.

(1) This paper makes full use of the evaluation data of suppliers' contracts after compensation process.

(2) The method combines the data test and k-means algorithms. It can find the significant biased evaluation scores distribution. And then it can give a clustering by re-analysis the evaluation data.

Although the classification of the suppliers contracts performance is not sophisticated enough, but from the perspective of the product or research capacity of enterprises, it is enough. From the above empirical results, the performance of contracts that this enterprise still have a lot of room for improvement.

In fact, the method proposed in this paper can not only be applied to the evaluation of contracts performance, but also can be used in many other similar evaluation problems. In the next research, we will try to find the improvement direction.

\section{ACKNOWLEDGMENT}

This paper is supported by the National Natural Science Foundation of China (Grant No.71501007 \& 71672006). The study is also sponsored by the Aviation Science Foundation of China (Grant No.2014ZG51075) and the Technical Research Foundation.

\section{REFERENCES}

[1] J. A. Hartigan and M. A. Wong, A K-means clustering algorithm., Applied Statistics, vol. 28, pp. 100-108, 1979

[2] J. G. Liu and S. L. Xu, Applied research of weighted K-means algorithm in social networks, in International Forum on Computers, Electronics and Mechatronics, IFCEM 2014, August 27, 2014 - August 28, 2014, Zhuhai, China, 2014, pp. 286-290.

[3] N. Dhanachandra, K. Manglem and Y. J. Chanu, Image Segmentation Using K-means Clustering Algorithm and Subtractive Clustering Algorithm, in 11th International Conference on Communication Networks(ICCN2015), August 21, 2015 - August 23, 2015, Bangalore, India, 2015:764-771.

[4] A. Feizollah, N. B. Anuar, R. Salleh, and F. Amalina, Comparative study of k-means and mini batch k-means clustering algorithms in android malware detection using network traffic analysis, in 2014 4th International Symposium on Biometrics and Security Technologies, ISBAST 2014, August 26, 2014 - August 27, 2014, Kuala Lumpur, Malaysia, 2015:193-197. 
[5] Cornell, A., Five key turning points in the American space industry in the past 20 years: Structure, innovation, and globalization shifts in the space sector. Acta Astronautica, 2011. 69(11-12): 1123-1131.

[6] Yang, L., Key practices, manufacturing capability and attainment of manufacturing goals: The perspective of project/engineer-to-order manufacturing. International Journal of Project Management, 2013. 31(1): 109-125.

[7] Zhang Y. F., Luo Q. G. Comprehensive Rvaluation of Diesel Engine Performance for Armored Vehicles. Chinese Journal of Mechanical Engineering, 2006, 42: 205-208.

[8] Guo W. X., Xia Z. Q. and Wang H. X. Grey Relational Analysis Modelbased Variation Coefficient Weight for Evaluating Hydraulic Engineering Planning Schemes. In 2007 IEEE International Conference on Grey Systems and Intelligent Services, 2007:195-199.

[9] Abraham, A., et al., Application of Fuzzy Inference Techniques to FMEA, in Applied Soft Computing Technologies: The Challenge of Complexity. 2006, Springer Berlin Heidelberg. p. 161-171.

[10] Kai, M.T. On Fuzzy Inference System Based Failure Mode and Effect Analysis (FMEA) Methodology. Soft Computing and Pattern Recognition, 2009. SOCPAR 09. International Conference of. 2009. Malacca.

[11] Galvao A F, Wang L. Efficient Minimum Distance Estimator for Quantile Regression Fixed Effects Panel Data[J]. Journal of Multivariate Analysis. 2015, 133(0): 1-26

[12] Raychaudhuri, S., Introduction to Monte Carlo simulation, Simulation Conference, 2008. WSC 2008. Winter, vol., no., pp.91,100, 7-10 Dec. 2008 